\title{
“FLEXO.IN-FORM”: PROCESOS INTEGRADORES EN ENVOLVENTES LAMINARES A FLEXIÓN ACTIVA
}

\section{“FLEXO.IN-FORM": INTEGRATING PROCESSES IN BENDING-ACTIVE LAMINARY ENVELOPES}

\author{
Gronda Ma. Luciana \\ CONICET, FADU-UNL \\ grondaluciana@hotmail.com
}

\section{RESUMEN}

En este artículo se presenta un ejercicio experimental a Flexión Activa (FA): "Flexo.In-Form", cuyo objetivo consiste en utilizar el comportamiento a la flexión como herramienta de diseño a partir del trabajo aplicado a estructuras que basan su geometría en la deformación elástica de elementos inicialmente planos. La FA se define como la instrumentalización de una de las -"nuevas"capacidades inscriptas en la Materialidad Digital (MD) desde un Enfoque de Diseño Orientado al Desempeño (EDOD) y no como una tipología estructural predefinida. La propuesta metodológica se fundamenta en el "form-finding", analógico-digital, como un EDOD. En términos instrumentales, el modelado paramétrico - de lógica matemático algorítmica-, la simulación digital -para la predicción del comportamiento físico-, la evaluación de cálculo numérico computacional, y finalmente, la fabricación CNC operan reuniendo las instancias de ideación, simulación, análisis y fabricación desde una lógica continua que re-in-forma el modelo a partir de los datos obtenidos en cada momento. En este sentido, la experimentación empírico-analítica es amplificada digitalmente mediante Procesos Integradores (PI) que constituyen nuevos procedimientos y formas de abordaje para repensar los procesos de ideación y abren múltiples posibilidades para emancipar del paradigma moderno la condición material en arquitectura.

\section{ABSTRACT}

This article presents an experimental exercise to Bending-Active (FA): "Flexo.In-Form", whose objective is to use flexion behavior as a design tool based on work applied to structures that base their geometry on the elastic deformation of initially flat laminar elements. In this sense, the AF is presented as the instrumentalization of one of the - "new" - capacities inscribed in the Digital Materiality (MD) from a Performance-Based Design Approach (EDOD) - and not as a predefined structural typology and opens possibilities for the design of curved architectural envelopes. The methodological proposal is based on the "form-finding", analog-digital, as an EDOD. In instrumental terms, parametric modeling -of algorithmic mathematical logic-, digital simulation -for the prediction of physical behavior-, the evaluation of computational numerical calculation, and finally, CNC manufacturing operates by gathering the instances of ideation, simulation, analysis and manufacturing from a continuous logic that feeds back and informs the model from the data obtained at each moment. In this sense, empiricalanalytical experimentation is digitally amplified through Integrative Processes (IP) that constitute new procedures and approaches to rethink ideation processes and open multiple possibilities to emancipate - from the modern paradigm - the material condition in architecture.

Palabras Clave: Desempeño, "Form-finding", Diseño Paramétrico, Simulación física, Fabricación Digital.

Key Words: Performance, Form-Finding, Parametric Design, Physics Simulation, Digital Fabrication. 


\section{Artículo RECIBIDO: 18/9/19 | Artículo ACEPTADO: 25/10/19}

\section{INTRODUCCION}

Este artículo presenta la conceptualización de algunas reflexiones obtenidas del proceso de diseño y fabricación de Flexo.In-Form, un ejercicio de experimentación aplicada enmarcado dentro del propio proceso de la Tesis Doctoral en curso, la cual propone un aporte doble, con objetivos teóricos y aplicados.

En este sentido, la primera instancia conceptual se ha concentrado en definir el presente histórico de la disciplina en función de tres grandes giros de lo digital en arquitectura y en proponer categorías analíticas: Materia Viva; Materia Responsiva y Materia Activa (MA), en base a la sistematización obtenida del análisis de los casos de estudio inscriptos en el contexto teórico denominado: Materialidad Digital (MD). Dicha primera instancia conceptual parte de una hipótesis guía que afirma que, es imposible revertir las secuelas de la modernización más cruel sin un cambio radical de paradigma. Al tiempo que, habilitada por las "nuevas" capacidades materiales que admite la MD, estamos asistiendo a una transformación emergente de la condición material en arquitectura -de los modos de concebirla y de operar en ella-, la cual ha sido sistematizada, conceptualizada y explicada en publicaciones anteriores (Gronda y Chiarella, 2017).

Por otro lado, la segunda instancia de validación práctica de la Tesis Doctoral se recorta y ajusta a la factibilidad detectada en el propio contexto próximo de producción a los fines de convertir de analítica a operativa alguna de las categorías propuestas y concretamente operar en ella en términos aplicados. Estudios previos determinaron que, dentro de las 'nuevas' capacidades materiales contenidas en la categoría analítica de la MA, la Flexión Activa (FA) reúne ampliamente las condiciones de viabilidad determinantes para la concreción de la instancia de validación práctica a partir de la experimentación directa con herramientas de fabricación CNC (corte 2D) y materiales laminares estandarizados y ampliamente comercializados, por lo tanto, viables al contexto próximo de producción. Sobre la base del análisis de las posibilidades de acceso a los materiales, métodos e instrumentos, la instancia aplicada se concentra en dar respuesta a la hipótesis central de que operar en la MD exige abordar Procesos Integradores ("Integrative Approach", Lienhard et al. 2013) de ideación arquitectónica y se apoya en los fundamentos teóricos-metodológicos de la FA. En este sentido, el presente artículo constituye una continuación del trabajo presentado en el IX Congreso Regional de Tecnología de la Arquitectura (CRETA) y publicado por la Revista Arquitecno no10 (Gronda, Chiarella y Veizaga, 2017).

Flexo.In-Form pretende producir una aproximación a la MD en arquitectura como propuesta alternativa que desafía métodos 'convencionales' de diseño y construcción en arquitectura mediante el desarrollo de un algoritmo de diseño paramétrico orientado a optimizar la relación operacional entre: geometría, material y estructura en envolventes laminares curvas a FA.

\section{Contextos próximos de producción}

Las decisiones en torno a la implementación de determinada tecnología en arquitectura, a diferencia de la década anterior, hoy no refieren a la potencialidad de ampliar las capacidades creativas y productivas, si bien éste continúa siendo un aspecto fundamental, el desafío actual es orientar el diseño a la optimización de los recursos, materiales y energéticos. La optimización no tiene como trasfondo una visión positivista de la realidad, sino que posiciona a la sustentabilidad como paradigma de diseño. La simulación digital y las variables cuantitativas obtenidas de la evaluación numérico computacional promueven en la actualidad un proceso que, por su eficiencia, se acerca a lo 
sustentable mediante un EDOD o "Performance" (Gronda y Chiarella, 2017, p.2) que permite pensar nuevas simbiosis entre diseño arquitectónico, tecnología, entorno construido y medio ambiente.

El actual contexto de la IV Revolución Industrial nos sitúa en un momento especial de desarrollo y progresiva democratización tecnológica, aun así, en Latinoamérica y más precisamente en Argentina, los avances convergentes a la línea de investigación propuesta continúan concentrándose en las disciplinas y/o industrias mejor capitalizadas, en trabajos de diseño industrial y en la producción de objetos seriados, postergándose su uso en arquitectura por varias razones. En primer lugar, debido al alto grado de producción artesanal que mantiene su inercia tanto por cuestiones socioeconómicas vinculadas a los elevados costos de capacitación de la mano de obra requerida, como así también a factores culturales fuertemente arraigados a la tradición histórica de la disciplina; y por otro lado, la falta de desarrollo científico acorde a estos temas, vinculada la necesidad de mayor financiamiento para abordar, producir y montar experimentaciones materiales a escala arquitectónica. En este sentido, el problema de la escala resulta sin duda lo que más limita a la disciplina arquitectónica en cuanto a desarrollo científico aplicado y amerita trabajos que se aborden científicamente en esta dirección. Por lo tanto, se entiende que el aporte de este trabajo es particularmente relevante, siendo su principal objetivo el de experimentar el diseño desde PI operando a través de la asimilación de los enfoques, métodos, instrumentos y materiales propuestos por la MD.

\section{Contextos teóricos y metodológicos}

Se parte de un presente histórico definido y caracterizado por los procesos recientes de asimilación disciplinar de las lógicas digitales como condición emergente -e innovadora- que potencia y enriquece las posibilidades instrumentales, metodológicas y técnico-culturales que implica la implementación de los PI de ideación y fabricación digital. En este contexto, las instancias de pre-figuración y representación son orientadas hacia una redefinición positiva al pensar las posibilidades de una transformación progresiva de los procesos de fabricación y construcción en arquitectura que obliga al diseño a salirse de cierto determinismo histórico vinculado con la autonomía del obsesivo control casi estilístico del objeto arquitectónico que ha estado durante décadas condicionado por las restricciones de la geometría euclidiana y ligado a los modos de producción del paradigma mecánico. Un modelo que ha ido configurando una concepción separatista, presente en las diferentes instancias contenidas -e institucionalizadas- dentro del proceso proyectual, el cual ha condicionado al material a un uso pasivo, con tipologías y disposiciones espaciales y estructurales predefinidas y estandarizadas. Se pretende, entonces, por un lado, revertir la aplicación del paradigma separatista mecánico presente en la división metodológica de los procesos "convencionales" de proyectación (representación, cálculo y construcción) y, por otro lado, proponer alternativas a los modos de producción del mundo material derivados del modelo industrial, como lógicas hegemónicas en virtud de su incuestionable capacidad de reproducción técnica y económica pero que han llevado al límite cuestiones creativas, ambientales y sociales.

\section{Flexión Activa: La flexión como herramienta de diseño.}

Flexión activa se propone como un enfoque de diseño y no como una tipología estructural predefinida, consiste en la instrumentalización de la deformación elástica como propiedad del material para estabilizarse y auto-conformarse (Lienhard, 2014). El trabajo aplicado a estructuras que basan su geometría en la deformación elástica-activa de elementos laminares inicialmente planos abre nuevas complejidades para el proceso de diseño y construcción de envolventes arquitectónicas curvas orientadas a optimizar recursos. La instrumentalización se realiza mediante el EDOD y las técnicas empírico-analíticas del "form-finding", analógico-digital, como herramienta metodológica capaz de optimizar la relación entre: los esfuerzos estructurales activos, el rendimiento material y el diseño geométrico. 
Si bien la máxima tradicional en ingeniería es limitar la cantidad de curvaturas en las estructuras, esta tipología realmente utiliza la flexión para la creación de diseños curvos, complejos y extremadamente livianos. Avances previos dan cuenta de que esta lógica proyectual puede brindar aportes confiables para envolventes arquitectónicas laminares a través de la interacción de variables relacionales parametrizadas (La Magna, Schleicher and Knippers, 2016)

\section{Procesos Integradores (De Diseño, Simulación, Análisis y Fabricación Digital)}

Hace referencia a la lógica proyectual contenida en los conceptos, métodos e instrumentos que sugiere la MD. A partir de la incorporación de los PI el diseño logra emanciparse de la aplicación pasiva del paradigma mecánico. El material deviene in-formado y dinámico en base al estudio de las particularidades intrínsecas del material, y a los fines de potenciarlo en todas sus escalas y propiedades. Reposicionan a los diseñadores en un papel estratégico para la construcción de modelos científicos, fórmulas paramétricas o algoritmos de diseño in-formados con los datos obtenidos de un proceso de lógica continua e iterativa. Redefinen el rol del arquitecto en el proceso mismo de la búsqueda de la forma, amplían el campo de acción y exigen considerar simultáneamente, y sin jerarquías, las diferentes dimensiones o variables que operan en ella. A los fines de hacer especial foco en la propuesta metodológica para la elaboración de estrategias de diseño generalizables que respondan a la lógica de la FA y siendo el objetivo principal el de identificar las posibilidades de potenciar/optimizar la relación operacional entre tres variables fundamentales: los esfuerzos estructurales activos, el rendimiento material y el diseño geométrico, el ejercicio proyectual asumido suspende momentáneamente la pretensión de abordar una 'mirada totalizadora` propia a la noción disciplinar de proyecto en arquitectura. Cabe mencionar que todas las otras variables que bien podrían sumarse a la parametrización, que operan en la configuración del mundo tangible y que remiten directamente a decisiones proyectuales en arquitectura son momentáneamente postergadas.

\section{Del modelo didáctico pedagógico al prototipo fabricable 1:1: Flexo.In-Form}

La construcción del prototipo experimental Flexo.In-Form tuvo varias etapas de desarrollo que marcaron su evolución desde un modelo didáctico pedagógico, presente en la propuesta para el Módulo: Laboratorio de Representación e Ideación: RILAB-2018 (Chiarella, Gronda y Veizaga, 2019), hacia un modelo científico, en el sentido de la exigencia en rigurosidad y precisión, para su fabricación en escala 1 en 1, cuyo montaje fue asumido dentro del Módulo: Proyectación y Fabricación Digital: PyFD-2019, siendo ambas propuestas académicas de la Maestría en Arquitectura FADU-UNL.

\section{METODOLOGÍA}

La investigación propone utilizar el comportamiento a la flexión como herramienta de ideación para la experimentación formal y analizar las posibilidades de ampliar el espacio de diseño a partir del trabajo aplicado a estructuras que basan su geometría en la deformación elástica-activa de elementos laminares inicialmente planos. En el marco de dicho trabajo, se identifican materiales, instrumentos, métodos y enfoques que avanzan en rigurosidad y precisión técnica, a la vez que constituyen nuevos procedimientos y formas de abordaje que presentan potencial para repensar los procesos de ideación en arquitectura y abren múltiples posibilidades para emancipar la condición material mediante la instrumentalización de un nuevo nivel de integración en diseño.

Para el desarrollo de Flexo.In-Form se procede, en primer lugar, al diseño de un algoritmo con modelado paramétrico y simulación digital orientado a la fabricación de envolventes laminares curvas a flexión activa. Inicialmente, las cintas planas se flexionan/curvan mediante el motor de simulación física (Kangaroo) para obtener envolventes estructurales de auto-formación y luego se desenrollan 
para habilitar su fabricación con herramientas CNC. Este procedimiento permite, por un lado, lograr la eficiencia estructural desde el EDOD contenido en la aplicación de la lógica de la FA, mientras que, abre el espacio de diseño y construcción de superficies de forma libre ("free-form") continuas a partir de elementos de curvado simple (desarrollables) mediante un proceso de construcción muy sencillo y económico.

El trabajo aplicado a envolventes laminares utiliza placas rígidas que presentan posibilidades de deformación limitadas frente a los esfuerzos de flexión, ya que se deforman principalmente a lo largo del eje de inercia más débil y, por lo tanto, no pueden adaptarse fácilmente a geometrías complejas o de forma libre. Tomando esta limitación geométrica como una ventaja especial en términos de diseño, se instrumentaliza la deformación elástica del material potenciándolo a partir del diseño de su geometría, tanto con procesos de discretización geométrica en la instancia de ideación, como así también, mediante cortes estratégicos con maquinaria CNC para su fabricación. De esta forma, los prototipos construidos permiten corroborar empíricamente las fuerzas a las que puede ser sometido el material. Dichas limitaciones se integran como datos de diseño en la definición paramétrica diseñada específicamente (Grasshopper) y se utilizan para calibrar las simulaciones digitales de los esfuerzos físicos a la flexión (Kangaroo). El desempeño de la estructura se optimiza a partir del reajuste de su geometría, garantizando que las tensiones permanezcan dentro del rango de trabajo permitido por el material. La sincronización de los estudios físicos y digitales es la que debe garantizar que las técnicas de búsqueda de formas proporcionen una descripción precisa del comportamiento real del material y, al mismo tiempo, logren brindar una mayor retroalimentación sobre la geometría resultante de la estructura.

El "form-finding" analógico es la determinación escalar de la forma a través de la replicación de las condiciones materiales y contextuales del mundo real, constituye un método bien establecido en arquitectura e ingeniería que apunta a optimizar la geometría respecto al comportamiento material y su desempeño estructural. Y si bien, hay una larga historia de experimentación en este campo apoyada por investigaciones científicas y ejemplos construidos incluso antes del uso generalizado del diseño computacional en arquitectura, aun así, continúa aportando una lectura esencial para la innovación e investigación en diseño. (Gronda, Chiarella, Veizaga, 2017, p.50). En este sentido, el form-finding analógico-digital (como estrategia metodológica de aproximación y búsqueda de la forma optimizada) declara cierta continuidad en los modos de concebir la forma compleja, pero avanza en términos instrumentales, digitalizando una práctica disciplinar histórica a partir de $\mathrm{PI}$.

\section{DESARROLLO: PI de Diseño y Fabricación en Flexo.in-form}

\section{Diseño}

Desde un proceso paramétrico, se desarrolló un algoritmo en la plataforma Grasshopper (dentro de la aplicación Rhinoceros 3D) y se utilizaron los plug-in Kangaaro y Karamba3D para la simulación física en tiempo real. El algoritmo construye inicialmente un hexágono dentro de un triángulo base, cuyos vértices se pueden adaptar a cualquier topología. A partir de la polilínea hexagonal descrita, se individualizan los 6 segmentos que la constituyen y con un "pattern false/true" se identifican las 3 líneas de anclaje y las 3 líneas de flexión. Mediante la división de las 3 líneas de anclaje en segmentos iguales se identifican los puntos de apoyo, que conectándolos con los puntos de los otros dos apoyos generamos la grilla triaxial base que conforma las cintas del modelo como mallas trianguladas a partir de los vértices de las intersecciones entre las líneas de la grilla. El orden topológico en la descripción paramétrica es lo que permite independizar las mallas para su evaluación física y garantiza el contacto entre los bordes de las cintas adyacentes y caras coplanares donde se cruzan las cintas (Figura 1). 


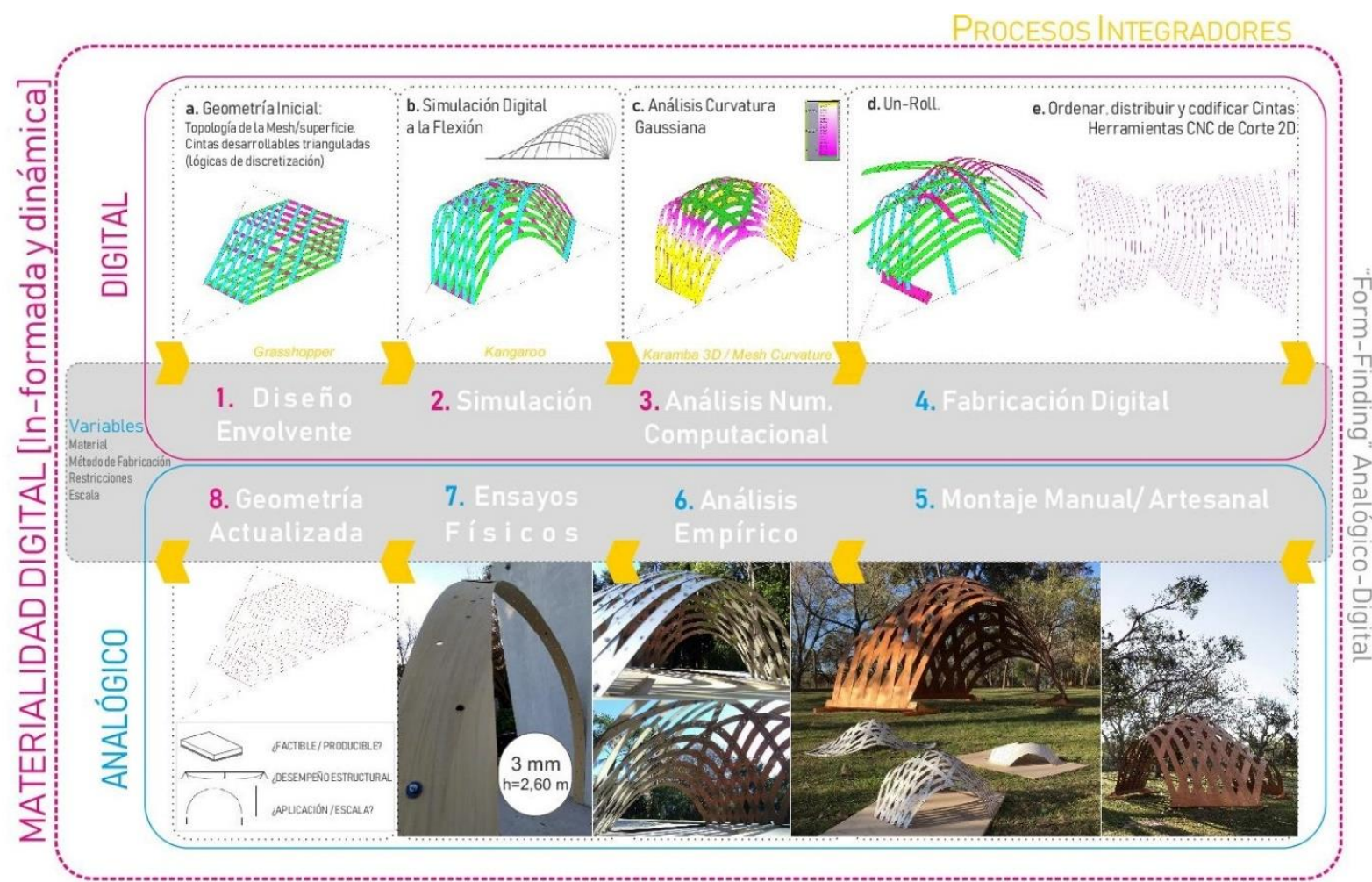

Figura 1: Procesos Integradores de diseño y construcción para envolventes laminares a Flexión Activa. Fuente: Elaboración propia.

\section{Simulación}

En un proceso de "form-finding" digital, se aplica una fuerza "Z" a las cintas generadas y fijándolas a los bordes de apoyo se flexionan en relación directa a la intensidad de la fuerza y a la resistencia a la flexión del material (variables o datos relacionales), formando una trama geométrica estructural de componentes laminares y curvatura simple que pueden desarrollarse para su fabricación.

Con el componente de simulación física Karamba se simuló y analizó la deformación del modelo y la tensión del material flexionado permitiendo calibrar el espesor del material a utilizar en función de los rangos admisibles (máximos y mínimos) (Figura 2).
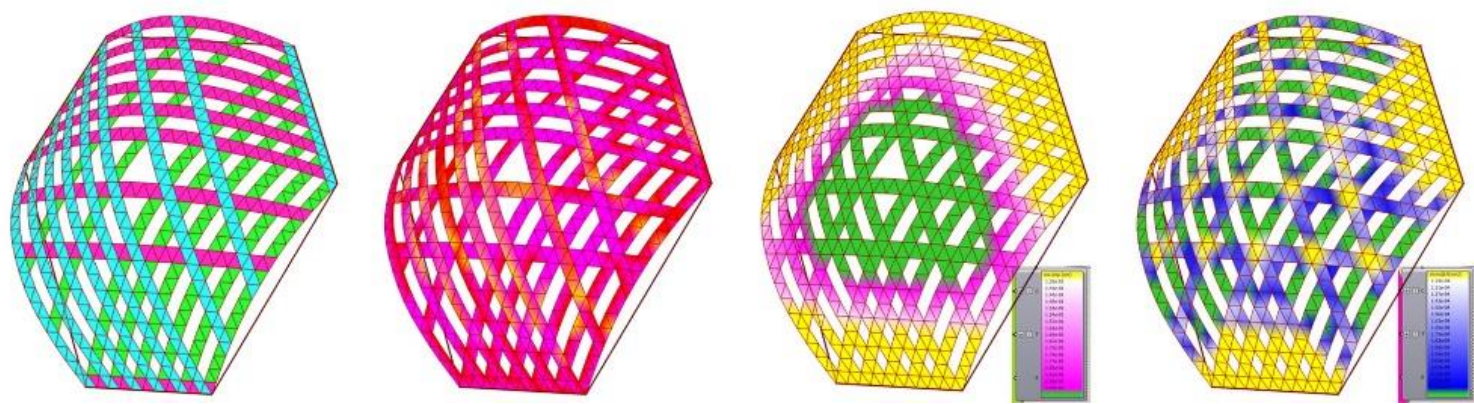

Figura 2: Simulación a flexión, Análisis de Curvatura Gaussiana y Van Mises Stress. Fuente: Elaboración propia. 


\section{Análisis numérico computacional}

La curvatura gaussiana, por un lado, analiza las zonas de la envolvente con doble curvatura permitiendo controlar, dentro del rango admisible del material, la torsión que supone en las cintas. El análisis del radio de curvatura máximo y mínimo identifica el componente, y el sector del componente, más y menos curvado y adapta la geometría a los rangos admisibles y/o deseables para la flexión del material.

\section{Fabricación digital}

El proceso de fabricación exige considerar el problema de la escala desde las instancias iniciales de ideación, implica atender al tamaño de los componentes digitales, a las medidas comerciales de los materiales laminares disponibles en el mercado y al tamaño máximo de corte viable en función de la herramienta CNC seleccionada para la producción. En un proceso integrador CAD/CAM la definición paramétrica asocia todas las acciones y restricciones de diseño en relación a la escala para la fabricación. El componente "Un-Roll" de Kangaaro desenrolla las cintas, luego el "Orient" las abate al plano X, Y; "Split" las fragmenta en partes cuyo máximo es el tamaño de la mesa de corte y "Rotate" gira las partes en el sentido de la fibra del material utilizado, otorgando continuidad de resistencia elástica a flexión en toda la cinta, luego "Nesting" las anida minimizando los desperdicios materiales.

Se ensayaron dos modelos en karical 0.08 y $0.06 \mathrm{~mm}$ en escalas $1: 10$ y 1:5 testeando la relación más conveniente entre geometría, material y comportamiento. Pero dichos prototipos experimentales no eran aun lo suficientemente representativos para el paso a la escala 1:1, principalmente en relación a la estabilidad de la forma global. En el cambio de escala es cuando la posibilidad de reajuste permanente del modelo digital se vuelve relevante. La asociación paramétrica de las variables geométricas, las propiedades y espesor del material y el desempeño del conjunto evaluado digitalmente, van sugiriendo los ajustes necesarios para afrontar el cambio de escala.

Finalmente, siendo el contrachapado altamente idóneo para grandes deformaciones a flexión por su relativo bajo módulo elástico, el modelo digital en escala 1:1 se ajustó para ser fabricado en multilaminado fenólico de $3 \mathrm{~mm}$.

\section{Montaje manual / artesanal ${ }^{1}$}

El montaje también implicó el abordaje del problema de la escala, no de las dimensiones de los componentes, de los materiales y las herramientas, sino de su peso, cantidad de componentes y de los recursos humanos necesarios. Siendo la instancia más analógica del proceso, para el montaje se realizó un instructivo detallado con la ubicación y el ensamble de los componentes de cada cinta con

\footnotetext{
${ }^{1}$ Sobre experiencia del montaje de Flexo.In-Form se sugiere ver: Gronda, María Luciana, Mauro Chiarella y Martín Veizaga. «Flexo.In-Form.2019. Manufactura digital y optimización geométrica mediante programación visual». Polis, n 16 (2019). https://www.fadu.unl.edu.ar/polis/flexo-in-form-2019-manufactura-digital-y-optimizacion-geometricamediante-programacion-visual/
} 
la debida codificación. El proceso de "izamiento" o conformación a la flexión del prototipo se realizó en aproximadamente 3 horas y con un grupo de 25 alumnos de la maestría.

El montaje de Flexo.In-Form o "levantamiento" del prototipo en escala 1:1 exhibió todos los temas abordados en las etapas anteriores: diseño; simulación; análisis y fabricación, como así también, cada una de las variables relacionales: geometría; material; estructura; resistencia; peso; manipulación y desempeño, otorgando conclusiones que formarán parte de futuras parametrizaciones para alcanzar mayor precisión o rigor científico, pero, fundamentalmente, se validó la utilidad de los PI como propuesta metodológica para el reajuste de un próximo modelo/prototipo.

\section{DISCUSIÓN DE RESULTADOS Y CONCLUSIONES}

El diseño, construcción y montaje de Flexo.In-Form permitió analizar las ventajas estructurales que pueden ser generadas mediante la deformación elástica activa en envolventes laminares a FA habilitando construir una mirada crítica sobre el avance de los procesos computacionales en arquitectura. Sobre la base de las estrategias formales e investigación proyectual con PI, la construcción de un modelo "científico" de simulación digital aporta vistas previas confiables para envolventes arquitectónicas curvas a través de la interacción de variables relacionales parametrizadas, lo que permite una mayor precisión a la hora de seleccionar y definir los materiales, las formas, los espacios y las tecnologías, minimizando así las decisiones basadas únicamente en la definición de categorías tipológicas estructurales, tradición local o experiencia directa. La experiencia verifica que la metodología propuesta como EDOD permite orientar los procesos de diseño a la optimización de los recursos. La integración estratégica de modelos de simulación físico-geométrica sumada a la herramienta metodológica del form-finding, analógico-digital, amplía el espacio de diseño y las posibilidades de construcción de envolventes laminares curvas mediante la discretización geométrica para la fabricación de componentes complejos a partir de materiales laminares ampliamente disponibles en el mercado.

El mayor reto es dominar los datos de la simulación para cuantificar, analizar y añadir el esfuerzo a las tensiones de flexión en el cálculo estructural para contribuir a un modelo científico cada vez más riguroso, tanto de la forma global como de las características locales de los componentes constitutivos de las cintas, en las cuales, las características geométricas y las propiedades del material son inevitablemente relacionales y afectan al desempeño estructural. 


\section{REFERENCIAS}

Gronda, Luciana; Chiarella, Mauro y Veizaga Martín. (2019). "RILAB - architectural envelopes From spatial representation (generative algorithm) to geometric physical optimization (scientific modeling)". In: Sousa, JP, Xavier, JP and Castro Henriques, G (eds.), Architecture in the Age of the 4th Industrial Revolution - Proceedings of the 37th eCAADe and 23rd SIGraDi Conference - Volume 3, University of Porto, Porto, Portugal, 11-13 September 2019, pp. 1724. Disponible en: http://papers.cumincad.org/cgi-bin/works/Show?ecaadesigradi2019_249

Gronda, Luciana; Chiarella, Mauro y Veizaga Martín. (2017). "Diseño orientado a la optimización en envolvente laminares de flexión activa". Revista ARQUITECNO X. Ediciones ITDAHu. Resistencia. vol. 1 p. 48-57. ISSN 0328-0896.

Gronda, Luciana; Chiarella, Mauro. (2017). "Materialidad Digital. Análisis de estrategias performativas transferibles al diseño resiliente". En: SIGraDi 2017 [Proceedings of the 21th Conference of the Iberoamerican Society of Digital Graphics - ISBN: 978-956-227-439-5] Chile, Concepción 22 - 24 November 2017, pp.51-59. Disponible en: http://papers.cumincad.org/cgibin/works/Show?sigradi2017_007

La Magna, Riccardo; Schleicher, Simon and Knippers. Jan. (2016) "Bending-Active Plates. Form and Structure". In: Riccardo La Magna, Jan Knippers S. Adriaenssens, F. Gramazio, M. Kohler, A. Menges, M. Pauly (eds.): Advances in Architectural Geometry. () vdf Hochschulverlag AG an der ETH Zürich, DOI 10.3218/3778-4, ISBN 978-3-7281-3778-4. Pp. 170-186. Disponible en: http://vdf.ch/advances-in-architectural-geometry-2016.html

Lienhard, Julian. (2014). Bending Active Structures, Form-Finding Strategies Using Elastic Deformation In Static And Kinematic Systems And Structural Potential Therein, Ph.D. Thesis, ITKE, University Of Stuttgart.

Lienhard, Julian (et al). (2013). "Active Bending, A Review on Structures where Bending is used as a Self-Formation Process". In: International Journal of Space Structures. Diciembre. DOI: 10.1260/0266-3511.28.3-4.187. Disponible en: https://www.researchgate.net/publication/261596411 